\title{
Using Social Media to Identify Events
}

\author{
Xueliang Liu \\ EURECOM \\ Sophia Antipolis, France \\ xueliang.liu@eurecom.fr
}

\author{
Raphaël Troncy \\ EURECOM \\ Sophia Antipolis, France \\ raphael.troncy@eurecom.fr
}

\author{
Benoit Huet \\ EURECOM \\ Sophia Antipolis, France \\ benoit.huet@eurecom.fr
}

\begin{abstract}
We present a method to automatically detect and identify events from social media sharing web sites. Our approach is based on the observation that many photos and videos are taken and shared when events occur. We select 9 venues across the globe that demonstrate a significant activity according to the EventMedia dataset and we thoroughly evaluate our approach against an official ground truth obtained directly by scraping the event venues' web sites. The results show our ability to not only detect events with high accuracy but also mine and identify events that have not been published in popular event directories such as Last.fm, Eventful or Upcoming. In addition to the textual identification of events, we show how we can build visual summaries of past events providing viewers with a more compelling feeling of the event's atmosphere.
\end{abstract}

\section{INTRODUCTION}

Today's mobile devices and network infrastructures enable users to easily capture and distribute rich multimedia content wherever they are located. Hence, it is becoming common for people to capture and share images or videos of their every day life using their mobile phones or digital camera. A plethora of niche mobile applications such as instagr.am ${ }^{1}$ or color ${ }^{2}$ are connected to large social media applications such as Flickr, YouTube and Facebook and contribute to the exponential growth of social media data available online. How to leverage the explosion of this vast amount of data to benefit web users at large is, however, still an open and challenging problem.

In this paper, we address the problem of structuring social media activity into events and in particular how to automatically detect those events and their properties (location, time and participation). Event directories such as Last.fm, Eventful and Upcoming publish information about scheduled events. Users benefit from this information for tagging

\footnotetext{
${ }^{1}$ http://instagr.am/

2 http://color.com/
}

Permission to make digital or hard copies of all or part of this work for personal or classroom use is granted without fee provided that copies are not made or distributed for profit or commercial advantage and that copies bear this notice and the full citation on the first page. To copy otherwise, to republish, to post on servers or to redistribute to lists, requires prior specific permission and/or a fee.

WSM'11, November 30, 2011, Scottsdale, Arizona, USA.

Copyright 2011 ACM 978-1-4503-0989-9/11/11 ...\$10.00. and structuring multimedia material in order to ease their future retrieval. While such services are becoming increasingly popular, they are often incomplete, sometimes inaccurate in terms of the information they provide and always working as silos that lock information into the sites. On the other hand, they largely overlap in terms of coverage and generally fail to give an indication of users' experience of an event's atmosphere, while this feature is considered as of primary importance to support users in deciding to attend or not an upcoming event. In contrast, one can find many photos shared online for specific events. This activity may be analyzed in order to detect and identify events occurrence and provide attractive summary. The problem we tackle is therefore how can we make use of metadata attached to media and events (tags, description, geographic location) to create the missing link between an event description and its illustrating media documents.

The paper is structured as follows. In section 2, we briefly describe the dataset used as well as the LODE and Media Resource ontologies for representing descriptions of events and media scraped from social media web sites. In section 3 , we detail our approach for detecting and identifying events based on user activity in social media and we show how we find more media related to detected events in order to create their visual summaries. We present some related work in section 4 . Finally, we give our conclusions and outline future work in section 5 .

\section{DATA ACQUISITION}

Several web sites contain information about scheduled events, of which some may display media captured at these events. Using the public API offered by the web sites, several datasets containing event and media descriptions have been recently published $[2,14]$. In this section, we briefly present the EventMedia $^{3}$ dataset (section 2.1) and we describe how we have chosen 9 venues for validating our approach (section 2.2).

\subsection{EventMedia = Flickr meets Event Direc- tories}

We use part of the EventMedia dataset which is composed of more than 1,7 million photos explicitly associated to more than 110,000 events [14]. This dataset has been built from the overlap in metadata between four popular web sites, namely Flickr as a hosting web site for photos and Last.fm, Eventful and Upcoming as a documentation of past and upcoming events. Explicit relationships between sched-

${ }^{3}$ http://eventmedia.cwi.nl/ 
uled events and photos are looked up using special machine tags such as lastfm: event=XXX or upcoming: event=XXX.

Descriptions of events are scraped from those three event directories using their API and represented according to the LODE ontology ${ }^{4}$, which provides a minimal model that encapsulates the most useful properties for describing events [12]. The goal of this ontology is to enable interoperable modeling of the "factual" aspects of events, where these can be characterized in terms of the four Ws: What happened, Where did it happen, When did it happen, and Who was involved. Furthermore, the W3C Ontology for Media Resource ${ }^{5}$ is used to describe the media resources. This model provides properties for describing media properties such as the duration of a video, its target audience, copyright, genre, rating or the various renditions of a photo. This ontology contains a formal set of axioms defining mapping between different metadata formats for multimedia.

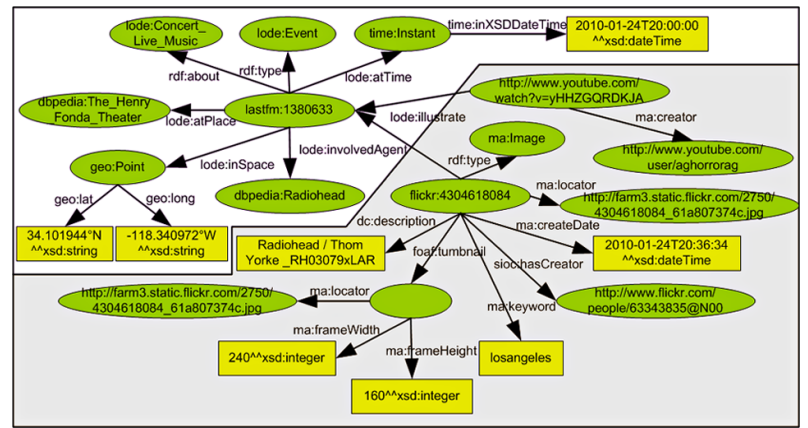

Figure 1: The Radiohead Haiti Relief Concert described with LODE (top) and illustrated with media described by the Media Ontology (bottom)

Figure 1 depicts the metadata attached to the event identified by 1380633 on Last.fm according to the LODE ontology. More precisely, it indicates that an event categorized as a Concert has been given on the 24th of January 2010 at 20:00 PM in the Henry Fonda Theater featuring the Radiohead rock band. The link between the media and the event is realized through the lode:illustrate property, while more information about the sioc:UserAccount can be attached to his URI. Hence, we see that the video hosted on YouTube has for ma:creator the user aghorrorag.

\subsection{Selecting Popular Venues}

The EventMedia dataset has knowledge of more than 13,000 different venues for which at least one description of event explicitly associated to at least one photo is available. From this very large dataset, we selected 9 venues that proved to have a significant activity the last three years. Table 1 shows the number of events, photos and distinct users for those venues during this period according to the EventMedia dataset ${ }^{6}$. The ranking value corresponds to the popularity of the venue in the entire dataset when the sorting criteria is the number of distinct users that have uploaded at least one photo on Flickr taken during an event hosted at those venues.

These 9 venues are diverse in terms of the type of event they generally host ranging from music concerts and festi-

\footnotetext{
4http://linkedevents.org/ontology/

${ }^{5}$ http://www.w3.org/TR/mediaont-10/

${ }^{6}$ SPARQL endpoint: http://semantics.eurecom.fr/sparql.
}

Table 1: Number of events, photos and distinct users for 9 venues in the EventMedia dataset

\begin{tabular}{|c|r|r|r|r|}
\hline Venue & NbEvents & NbPhotos & NbUsers & rank \\
\hline Melkweg & 352 & 6912 & 266 & 1 \\
\hline Koko & 151 & 3546 & 155 & 5 \\
\hline HMV Forum & 106 & 2650 & 130 & 8 \\
\hline $\begin{array}{c}111 \text { Minna } \\
\text { Gallery }\end{array}$ & 24 & 1369 & 105 & 14 \\
\hline $\begin{array}{c}\text { Hammersmith } \\
\text { Apollo }\end{array}$ & 79 & 2124 & 96 & 20 \\
\hline $\begin{array}{c}\text { Circolo } \\
\text { Magnolia }\end{array}$ & 79 & 2190 & 76 & 40 \\
\hline $\begin{array}{c}\text { Circolo } \\
\text { degli Artisti }\end{array}$ & 784 & 2590 & 86 & 43 \\
\hline Rotown & 204 & 3623 & 49 & 48 \\
\hline $\begin{array}{c}\text { Ancienne } \\
\text { Belgique }\end{array}$ & 212 & 7831 & 56 & 83 \\
\hline
\end{tabular}

vals, to circus or exhibitions. They are mainly situated in Europe (UK, The Netherlands, Italy, Belgium) except the venue 111 Minna Gallery which is located in San Francisco (USA). More importantly, for all these venues, it is possible to get a perfect ground truth of the events they host since they all publish an up-to-date program on their web site. We have developed different scrapers of these web sites in order to get accurate ground truth of past events ${ }^{7}$.

\section{DETECTING EVENTS BASED ON SOCIAL MEDIA ACTIVITY}

More and more media shared on the web are provided with latitude and longitude coordinates, either through user input or directly by the capture device itself. We also assume that when an event takes place, there will be enough persons taking picture or videos and later sharing them online. Our approach consists in measuring photo peaks at known venues in order to detect an event occurrence. It is therefore necessary to model the venue location.

\subsection{Venue Bounding-Box Location Estimation}

The Flickr API allows users to query photos based on geographical location. However, getting the geographical coverage or outer box of a place is not trivial. We address this issue by combining information from event and media directories. We retrieve events (EventID $=E$ ) that took place at a given venue (VenueID $=V$ ) using the Last.fm API. Then, we use the machine tags "lastfm:event $=E$ " to search for geotagged media on Flickr. For each venue, a bounding box is finally computed using the GPS coordinates of the retrieved photos (algorithm 1). The final bounding box is estimated as the minimized rectangle of the GPS coordinates after removing the outliers defined as the photos which are located further than twice the variance of the set in either direction (longitude or latitude).

Figure 2 shows the result of the bounding box estimation for the venue Melkweg (Amsterdam, NL). The blue marker is the GPS location of Melkweg according to Last.fm, while the red markers are the places where photos labeled with machine tags from past events have been shared on Flickr. The red rectangle corresponds to the learnt bounding box for the venue Melkweg. We see that some photos taken too far away from the venue have been appropriately discarded.

\footnotetext{
${ }^{7}$ Scrapers available at http://scraperwiki.com/profiles/Hou/.
} 

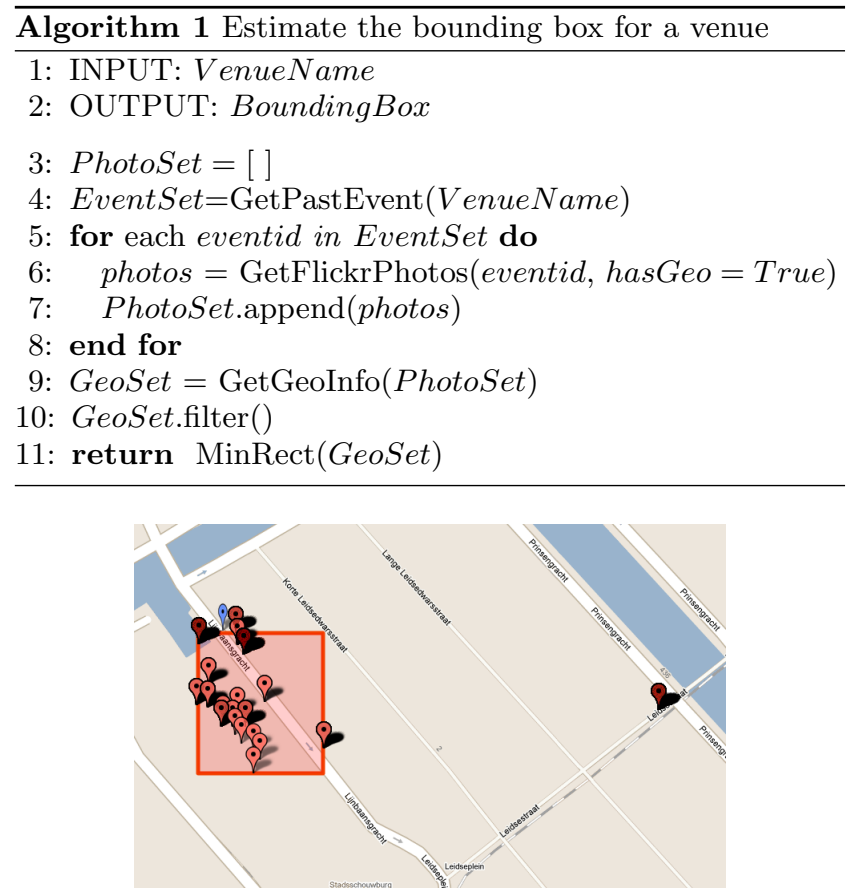

Figure 2: Bounding Box for the venue Melkweg in Amsterdam (NL)

We define the bounding boxes for the 9 venues selected in Table 1 and we crawl all photos taken at those locations during a one month period from May 1st 2010 to May 31st 2010. A collection of 4604 geo-tagged photos has been obtained. The number of geo-tagged photos hosted on Flickr represents only a tiny fraction of all photos shared online. In order to obtain more relevant photos, we also use the venue name as a query term for retrieving photos during the same period resulting in another 4589 photos (Table 2). Surprisingly, we notice that there are very few photos common to both queries. This shows that users seldom label their photos with a location name when geo-coordinates are available.

Table 2: Number of photos taken in the 9 selected venues during May 2010

\begin{tabular}{|r|r|r|r|r|r|}
\hline ID & Name & $\begin{array}{c}\text { Geotagged } \\
\text { Photos }\end{array}$ & $\begin{array}{c}\text { Venue Name } \\
\text { tagged Photos }\end{array}$ & Duplicate & Total \\
\hline 1 & Koko & 372 & 2040 & 3 & 2409 \\
\hline 2 & Rotown & 90 & 273 & 1 & 362 \\
\hline 3 & Melkweg & 363 & 700 & 8 & 1055 \\
\hline 4 & HMV Forum & 184 & 412 & 0 & 596 \\
\hline 5 & $\begin{array}{c}111 \text { Minna } \\
\text { Gallery }\end{array}$ & 937 & 3 & 0 & 940 \\
\hline 6 & $\begin{array}{c}\text { Ancienne } \\
\text { Belgique }\end{array}$ & 2206 & 288 & 2 & 2492 \\
\hline 7 & $\begin{array}{c}\text { Circolo } \\
\text { degli Artisti }\end{array}$ & 70 & 553 & 1 & 622 \\
\hline 8 & $\begin{array}{c}\text { Circolo } \\
\text { Magnolia }\end{array}$ & 95 & 236 & 0 & 331 \\
\hline 9 & $\begin{array}{c}\text { Hammersmith } \\
\text { Apollo }\end{array}$ & 287 & 84 & 0 & 371 \\
\hline
\end{tabular}

\subsection{Analyzing the Flickr Activity around Venues}

We are interested in detecting events by monitoring the social media sharing activity at specific locations. The 9178 photos gathered by crawling Flickr during May 2010 using

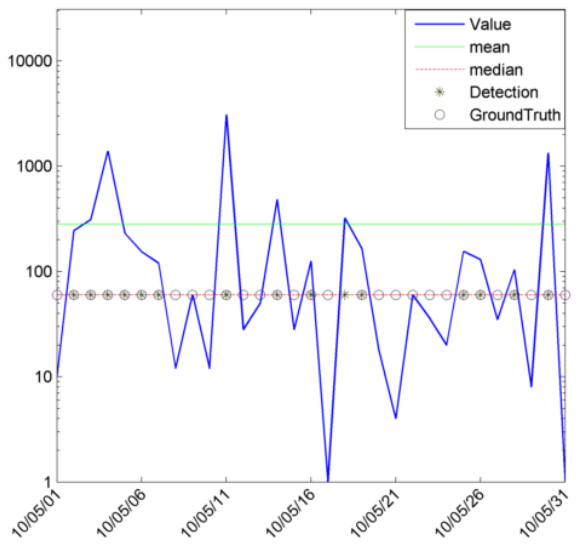

Figure 3: Event detection during May 2010 in the Melkweg (Amsterdam, NL)

either the geographical bounding boxes or the venues name will be the basis for the event detection experiments. Our objective is to identify the date and title of an event given its venue or location. Figure 3 depicts the daily number of photos taken at the Melkweg in May 2010 that have been shared on Flickr. One can see that the number of pictures taken varies temporally over the month. Our approach consist in carefully selecting the dates with peaks and consider those candidate date as events. More formally, let us consider the time series $\left\{d_{i}, i \in[1, T]\right\}$ that represents the temporal evolution of the number of photos taken at a given venue $v$. The event $e$ starting at time $t$ is detected when the number of photos shared is greater than a given threshold.

$$
e_{t}=\arg _{i}\left(t_{i}>\text { THRESHOLD }\right)
$$

In this experiment, we compare three characteristics. The first is simply the number of online photos available for a given location and date $d_{i}=\left\|p_{i}\right\|$. The second attempts to incorporate the social dimension of the event by accounting for the number of different photo owners $u_{i}$. The third characteristic combines the previous ones by pondering the number of photos with the number of different person that shared those photos $d_{i}=\left\|p_{i} * u_{i}\right\|$. The choice of the threshold is an important factor of the events detection performance our approach can achieve. Venues hosting events have very different size and popularity. It is therefore unlikely a common threshold would provide a good and generic performance. Here, we experiment with the average and median value of $d_{i}$ as threshold.

\subsection{Detection Results and Evaluation}

We run this event detection method on the nine selected venues. Figure 3 depicts results for the first venue (Melkweg): the main blue curve shows the product of the number of photos and the number of photo owners per day $d_{i}$, the green curve corresponds to the mean and the red one is the median of the blue curve. The stars indicate the date for which an event is detected (based on median thresholding). The ground truth is indicated using circles over the median. One can see that many events would be missed using the mean threshold while the median is able to capture many more events while keeping the false detection rate low.

In May 2010, a total of 242 events are reported from the 
official schedules of the nine selected venues, but only 150 of them have been announced on Last.fm (and none on Eventful and Upcoming). We evaluate the performance with respect to both this absolute ground truth from the official venues and from what event directories are aware of. Once an event start time is identified, we use the tags of the corresponding photos to deduce the topic of the event. All words from tags and titles of photos taken on that day are parsed and sorted by their occurrence. The top 15 keywords are kept to infer the topic of individual events. Detected events are manually matched with ground truth events based on date and title. Matching events are those sharing a common starting date and for which at least one non stop-word can be found in both the top 15 keywords list of detected events and the title of the ground truth events. Of the 150 Last.fm events, 136 can be matched to the ground truth data, using our starting date and title matching rule. When analysing our detection result, we identify 17 events that are not present in Last.fm but are present in the ground truth. In other words, our approach can automatically extend the event coverage of Last.fm with $10 \%$ new events. Table 3 shows the positive detection on the Melkweg (start time and tags). For each event, the ground truth from the official venue web site and from Last.fm are compared with the most relevant tags we detect.

Figure 3 indicates to favor the use of the median over the mean as threshold condition for detecting events. We have identified three characteristics upon which event detection could be performed: number of image, number of owners and number of image times number of owners. Table 4 gives the results for these 6 conditions. When the median is used for thresholding, more positive events are detected compared with the ones using the mean. Unfortunately, the number of false detection also increases. Of the three upload characteristics, the combined "Image*Owner" achieves the best overall performance with an F1-Measure of $\mathbf{0 . 2 8 9}$ with a median based threshold. Based on these results, we decide to use the median thresholding approach on the "Image*Owner" upload characteristic for event detection.

Table 4: Event detection on different conditions

\begin{tabular}{|c|c|c|c|r|}
\hline Source & Threshold & True Predict & False Predict & F1 \\
\hline \multirow{3}{*}{ Image } & mean & 43 & 21 & 0.211 \\
\cline { 2 - 5 } & median & 64 & 51 & 0.279 \\
\hline \multirow{3}{*}{ Owner } & mean & 56 & 56 & 0.246 \\
\cline { 2 - 5 } & median & 58 & 62 & 0.251 \\
\hline \multirow{2}{*}{ Image* Owner } & mean & 34 & $\mathbf{1 8}$ & 0.172 \\
\cline { 2 - 5 } & median & $\mathbf{6 7}$ & 53 & $\mathbf{0 . 2 8 9}$ \\
\hline
\end{tabular}

The overall detection results on the 9 venues are shown in Table 5. Since the detection of events is based on social media activity, any events for which no or too few photos have been shared on Flickr cannot be detected. The recall values reported in this table should therefore be interpreted with caution. As the current sharing trends continues to evolve though many platforms, we expect the number of "detectable" events using our proposed approach to grow accordingly. We also provide the number of events known by Last.fm for those venues during this period and we observe that our approach is able to correctly detect events unknown for this event directory (indicated with ${ }^{*}$ ).

\subsection{Illustrating Events with Media}

Event directories fail to provide viewers a good feeling
Table 5: Event detection results for the 9 selected venues

\begin{tabular}{|r|r|r|r|r|r|r|}
\hline \multirow{2}{*}{ VID } & \multirow{2}{*}{ GT } & \multicolumn{4}{|c|}{ Our Method } & \multirow{2}{*}{ LastFM } \\
\cline { 3 - 6 } & & Detect & Matched & $\mathbf{P}$ & $\mathbf{R}$ & 44 \\
\hline 1 & 69 & 15 & $\left(^{*}\right) 12$ & 0.800 & 0.174 & 0 \\
\hline 2 & 20 & 15 & $\left(^{*}\right) 8$ & 0.533 & 0.400 & 14 \\
\hline 3 & 14 & 12 & 9 & 0.750 & 0.643 & 0 \\
\hline 4 & 23 & 15 & $\left(^{*}\right) 2$ & 0.133 & 0.087 & 28 \\
\hline 5 & 38 & 15 & $\left(^{*}\right) 9$ & 0.600 & 0.237 & 13 \\
\hline 6 & 16 & 15 & 8 & 0.533 & 0.500 & 12 \\
\hline 7 & 22 & 15 & $\left(^{*}\right) 8$ & 0.533 & 0.364 & 11 \\
\hline 8 & 25 & 3 & 1 & 0.333 & 0.040 & 14 \\
\hline 9 & 15 & 15 & 10 & 0.667 & 0.667 & 136 \\
\hline total & 242 & 120 & 28 & 0.558 & 0.277 & \\
\hline
\end{tabular}

about the atmosphere of events [13]. Showing photos and videos related to past events is a natural way to provide more insight to viewers. We follow the approach proposed in [9] for mining and visually pruning media related to those detected events. Let $F$ be the set of photos shared on Flickr around the date of a predicted event $i$ ( 1 day before and 2 days after) containing keywords corresponding to the event title. Let $E$ be the set of photos explicitly linked with the event $i$ (using the event machine tag) from the EventMedia dataset. We select the images from $F_{j}$ whose visual similarity match closely one of the images in $E$ as additional representative media for an event. Visual similarity between images is computed as follows:

$$
L_{1}\left(F_{j}, E_{i}\right)=\sum_{k}\left|F_{j}(k)-E_{i}(k)\right|
$$

where $F_{j}(k)$ and $E_{i}(k)$ are normalized concatenating low level feature vector of the images (225D color moments in Lab space, 64D Gabor texture, and 73D Edge histogram). $F_{j}$ is added to the set of media illustrating the event when

$$
L_{1}\left(F_{j}, E_{i}\right)<T H D_{i}
$$

where $T H D_{i}$ is the threshold which is also learned from the $E$ data.

$$
T H D_{i}=\min _{\{j\} \backslash i} \sum_{k} \mid \text { EventMedia }_{j}(k)-\text { Event Media }_{i}(k) \mid
$$

In addition, if the owner of $F_{j}$ has shared more photos during this period, they are automatically added as illustrative media for the event.

For each of the 67 events detected, we query Flickr for photos containing event specific words $w_{e}$ and uploaded within a four day window around the event ( 1 day earlier and 2 days after). This results in a potential enrichment for 23 events (see Table 6). This table reports four different information about each individual event. The first part, "Detection Dataset", corresponds to the data used for detecting events and from which a subset of relevant photos will be used for creating the visual model later used for pruning. The second part, "Enriching Source", reports the number of new retrieved images which will be considered for enriching the event illustration set. The third part shows the visual pruning results. Finally, the last part gives the total number of photos that illustrate the event after enrichment and visual pruning.

Let us take the first event as an example. For this event, there are 101 photos taken on that day at this location, and 66 of them are relevant to the event (based on textual keywords match). 32 additional photos are obtained 
Table 3: Event Detection Results on Melkweg

\begin{tabular}{|c|c|c|c|c|c|c|}
\hline \multirow[b]{2}{*}{ Venue } & \multicolumn{2}{|c|}{ Detection Results } & \multicolumn{2}{|r|}{ Ground Truth (Official) } & \multicolumn{2}{|r|}{ LastFM } \\
\hline & Date & Tags & Date & $\begin{array}{c}\text { Title } \\
\end{array}$ & LastFM & Title \\
\hline melkweg & $03 / 05 / 2010$ & $\begin{array}{l}\text { parkwaydrive } \\
\text { drive parkway }\end{array}$ & $03 / 05 / 2010$ & $\begin{array}{l}\text { Parkway Drive / Despised Icon / } \\
\text { Winds Of Plague / The Warriors / } 50 \text { Lions }\end{array}$ & 1336473 & Parkway Drive \\
\hline melkweg & $02 / 05 / 2010$ & $\begin{array}{l}\text { flight conchords } \\
\text { flightoftheconchords }\end{array}$ & $02 / 05 / 2010$ & Flight Of The Conchords - UITVERKOCHT & 1439320 & $\begin{array}{l}\text { Flight of } \\
\text { the Conchords }\end{array}$ \\
\hline melkweg & $04 / 05 / 2010$ & flightoftheconchords & $04 / 05 / 2010$ & Flight Of The Conchords - UITVERKOCHT & 1439407 & $\begin{array}{l}\text { Flight of } \\
\text { the Conchords }\end{array}$ \\
\hline melkweg & $05 / 05 / 2010$ & $\begin{array}{l}\text { mayerhawtorne } \\
\text { mayer hawthorne }\end{array}$ & $05 / 05 / 2010$ & Mayer Hawthorne \& The County & 1416229 & $\begin{array}{l}\text { Mayer Hawthorne } \\
\text { The County }\end{array}$ \\
\hline melkweg & $11 / 05 / 2010$ & bonobo & $11 / 05 / 2010$ & Bonobo - UITVERKOCHT & 1398102 & Bonobo \\
\hline melkweg & $14 / 05 / 2010$ & paulweller paul & $14 / 05 / 2010$ & Paul Weller - UITVERKOCHT & 1406677 & Paul Weller \\
\hline melkweg & $18 / 05 / 2010$ & brokensocialscene & $18 / 05 / 2010$ & Broken Social Scene - UITVERKOCHT & 1334429 & Broken Social Scene \\
\hline melkweg & $19 / 05 / 2010$ & $\begin{array}{c}\text { mikestern } \\
\text { richardbona }\end{array}$ & $19 / 05 / 2010$ & $\begin{array}{l}\text { Mike Stern band with special guest Richard } \\
\text { Bona featuring Dave Weckl \& Bob Malach }\end{array}$ & & \\
\hline melkweg & $25 / 05 / 2010$ & beattimemelkweg & $24 / 05 / 2010$ & Beattime - The Kika Edition & & \\
\hline melkweg & $26 / 05 / 2010$ & beattime & $24 / 05 / 2010$ & Beattime - The Kika Edition & & \\
\hline melkweg & $28 / 05 / 2010$ & offcentre & $28 / 05 / 2010$ & $\begin{array}{l}\text { Off Centre - day } 3 \text { - night met Kode } 9 \\
\text { / Falty DL / Gold Panda / Kelpe }\end{array}$ & & \\
\hline melkweg & $30 / 05 / 2010$ & joannanewsom & $30 / 05 / 2010$ & $\begin{array}{l}\text { Joanna Newsom } \\
\end{array}$ & 1425481 & Joanna Newsom \\
\hline
\end{tabular}

on Flickr through the enrichment process. A manual visual check identified 31 of those images to be relevant to the associated event. After automatic visual pruning 20 photos are left, and all of them are relevant photos, indicating that the pruning process has discarded the non relevant photo from the enrichment set. There is a final total of $86(66+20)$ photos obtained to illustrate this events. A visual representation of this event is depicted in Figure 4.

In summary, for the 23 events, there are 1678 photos used for event detection, out of which 1138 are thought to be sufficiently relevant based on tag similarity to be used to model the events visually. The enriching Flickr search retrieved 632 new photos. 464 of those photos are relevant to event according to manual visual check. The automated visual pruning algorithm identifies 584 relevant photos, from which 417 are judged relevant through human inspection. The proposed approach is therefore able to populate illustrative sets of media representing particular events with a precision of 0.714 and recall rate 0.898 . In addition, the EventIDs featuring a $\left(^{*}\right)$ are events for which no entry can be found in Last.fm but which have been detected by our algorithm.

We generate visual clusters for each events using the method proposed in [15] (Figure 4). During the enrichment phase, we expect to bring more diverse photos into the collection. For example, the Figure 4 depicts the event 2, which is held in Hammersmith Apollo with the title iggy stooges. Figure $4(\mathrm{~A})$ is generated from the relevant photos from the detection set that corresponds to photos that are either geotagged or tagged with a venue name. Figure 4(B) shows the collection of images resulting only from our enriching and visual pruning method. We can clearly see the increased visual diversity of the scenes between the two sets. The final set of images illustrating the iggy stooges event will be composed of both sets.

\section{RELATED WORK}

While the earlier works aiming at event discovery were based solely on text analysis and essentially focused on news documents $[7,6]$, event detection in social media enables to take into account rich context. For example, [5] proposed a framework to fuse tag relevance with location information and visual cues, and aimed at mining patterns from them. In [8], Cao et al. tried to leverage the contextual information

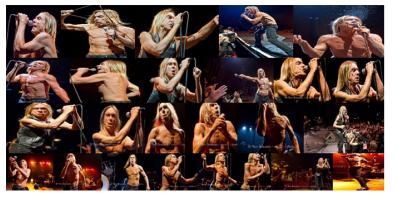

(A) Before enrichment

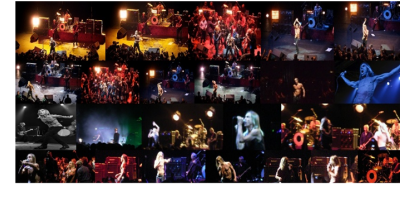

(B) After enrichment \& Pruning
Figure 4: Visual cluster for the event 2, which was held on 03/05/2010, in the venue Hammersmith Apollo with the title iggy stooges

of photos in social media to benefit the annotation process. First, event relation are mined from spatial and temporal metadata of photos, and then, the hierarchical structure is used to improve the simple annotation. In [1], the authors proposed a clustering framework to group images based on geographical coordinates and visual feature to depict different view of a location.

In [10], a framework to detect landmark and events to improve the user browsing and retrieval experience is proposed. The work presented in [4] attempts to identify public event using both spatial-temporal context and photo content. In [3], the authors exploit the social information from photos in Flickr in form of tags, titles and photo descriptions, for categorizing pictures into different event categories. In [11], the author proposed a framework to detect events based on the assumption that documents describing the same event will contain similar sets of keywords. In [2], Becker et $a l$. focused on identifying real-world events and their associated user-contributed social media documents automatically to enable event-based browsing and searching in state-of-theart search engines. Our work differs from these systems in that we combine rich semantic information and image analysis for detecting events in social media. Moreover, our event detection approach is not based on a user query but rather on monitoring media sharing behavior of users at particular location.

In some aspects, the work of Gao et al. [4] shows interesting complementarities with our work. The idea of employing Twitter data to search for additional information about an event could be used in our work to extend the set of relevant words describing events. As a result, the enrichment process would retrieve an even more diverse set of candidate images. 
Table 6: Illustrating events with photos

\begin{tabular}{|c|c|c|c|c|c|c|c|c|c|}
\hline \multirow[b]{2}{*}{ EventID } & \multirow[b]{2}{*}{ VenueID } & \multicolumn{2}{|c|}{ Prediction DataSet } & \multicolumn{2}{|c|}{ Enriching Source } & \multicolumn{3}{|c|}{ Pruning Results } & \multirow[b]{2}{*}{ Final Photo Nb } \\
\hline & & Photos & Related & Photos & Related & Photos & Precision & Recall & \\
\hline$(*) 1$ & 1 & 101 & 66 & 32 & 31 & 20 & 0.645 & 1 & 97 \\
\hline 2 & 9 & 44 & 43 & 48 & 48 & 48 & 1 & 1 & 91 \\
\hline 3 & 1 & 116 & 97 & 5 & 5 & 3 & 0.6 & 1 & 102 \\
\hline$(*) 4$ & 2 & 161 & 107 & 21 & 20 & 20 & 1 & 1 & 127 \\
\hline 5 & 2 & 61 & 17 & 1 & 0 & 1 & NULL & 0 & 17 \\
\hline 6 & 6 & 7 & 1 & 1 & 1 & 1 & 1 & 1 & 2 \\
\hline 7 & 1 & 238 & 201 & 4 & 0 & 2 & NULL & 0 & 201 \\
\hline$(*) 8$ & 2 & 95 & 44 & 9 & 9 & 9 & 1 & 1 & 53 \\
\hline 9 & 9 & 17 & 17 & 24 & 24 & 24 & 1 & 1 & 41 \\
\hline 10 & 6 & 4 & 3 & 5 & 5 & 5 & 1 & 1 & 8 \\
\hline 11 & 3 & 22 & 20 & 4 & 4 & 4 & 1 & 1 & 24 \\
\hline 12 & 3 & 3 & 2 & 5 & 5 & 4 & 0.8 & 1 & 7 \\
\hline 13 & 9 & 173 & 161 & 11 & 11 & 11 & 1 & 1 & 172 \\
\hline 14 & 5 & 70 & 37 & 5 & 5 & 4 & 0.8 & 1 & 42 \\
\hline 15 & 5 & 66 & 11 & 249 & 88 & 220 & 0.636 & 0.255 & 99 \\
\hline 16 & 9 & 9 & 2 & 1 & 1 & 1 & 1 & 1 & 3 \\
\hline$(*) 17$ & 2 & 135 & 53 & 14 & 14 & 14 & 1 & 1 & 67 \\
\hline 18 & 6 & 43 & 41 & 2 & 2 & 2 & 1 & 1 & 43 \\
\hline (*) $) 19$ & 2 & 95 & 78 & 4 & 4 & 4 & 1 & 1 & 82 \\
\hline 20 & 3 & 50 & 29 & 67 & 67 & 67 & 1 & 1 & 96 \\
\hline$(*) 21$ & 5 & 53 & 34 & 10 & 10 & 10 & 1 & 1 & 44 \\
\hline 22 & 5 & 52 & 11 & 54 & 54 & 54 & 1 & 1 & 65 \\
\hline 23 & 3 & 63 & 63 & 56 & 56 & 56 & 1 & 1 & 119 \\
\hline
\end{tabular}

\section{CONCLUSION AND FUTURE WORK}

We presented a novel approach for automatically detecting events and their properties (location, time and participation). The key idea of our contribution consists in temporally monitoring media shared on social web sites, registered to a specific location or referring to a specific venue. We show that using the appropriate thresholding function, it is possible to detect events in an efficient way. The result of our work can therefore be used to automatically structure online media. It was shown that the approach identified a number of events uncovered by the most popular event directories (Last.fm, Eventful and Upcoming). In addition, we have presented a process for automatically extending the number of photos illustrating an event. It consist in a two step process where first the image collection is extended through keyword search using the Flickr API before being pruned visually thanks to automatically created event visual models. The resulting photo montages better capture the atmosphere of the event. The EventMedia dataset can be further enhanced with more sources and more linkage. In particular, user participation at events using public Foursquare/Gowala check-in and live tweets can be added to enrich the overall knowledge one has about a past event. Our long term goal is to provide support for exploring and selecting events and associated media, and for discovering meaningful, surprising or entertaining connections between events, media and participants.

\section{REFERENCES}

[1] Y. Avrithis, Y. Kalantidis, G. Tolias, and E. Spyrou. Retrieving landmark and non-landmark images from community photo collections. In $18^{\text {th }}$ ACM International Conference on Multimedia, 2010.

[2] H. Becker, M. Naaman, and L. Gravano. Learning Similarity Metrics for Event Identification in Social Media. In $3^{\text {rd }}$ ACM International Conference on Web Search and Data Mining, 2010.

[3] C. Firan, M. Georgescu, W. Nejdl, and R. Paiu. Bringing order to your photos: event-driven classification of Flickr images based on social knowledge. In $19^{t h} A C M$
International Conference on Information and Knowledge Management, 2010.

[4] M. Gao, X.-S. Hua, and R. Jain. WonderWhat: Real-time Event Determination from Photos. In $20^{\text {th }}$ World Wide Web Conference, 2011.

[5] L. Kennedy, M. Naaman, S. Ahern, R. Nair, and T. Rattenbury. How flickr helps us make sense of the world: context and content in community-contributed media collections. In $15^{\text {th }}$ ACM International Conference on Multimedia, 2007.

[6] G. Kumaran and J. Allan. Text classification and named entities for new event detection. In $2^{\gamma^{t h}}$ International ACM SIGIR Conference, 2004.

[7] Z. Li, B. Wang, M. Li, and W.-Y. Ma. A probabilistic model for retrospective news event detection. In $28^{\text {th }}$ International ACM SIGIR Conference, 2005.

[8] C. Liangliang, L. Jiebo, H. Kautz, and T. Huang. Image Annotation Within the Context of Personal Photo Collections Using Hierarchical Event and Scene Models. IEEE Transactions on Multimedia, 11(2), 2009.

[9] X. Liu, R. Troncy, and B. Huet. Finding Media Illustrating Events. In $1^{\text {st }} A C M$ International Conference on Multimedia Retrieval, 2011.

[10] S. Papadopoulos, C. Zigkolis, Y. Kompatsiaris, and A. Vakali. Cluster-Based Landmark and Event Detection for Tagged Photo Collections. IEEE Multimedia, 18(1), 2011.

[11] H. Sayyadi, M. Hurst, and A. Maykov. Event Detection and Story Tracking in Social Streams. In $3^{\text {rd }}$ International AAAI Conference on Weblogs and Social Media, 2009.

[12] R. Shaw, R. Troncy, and L. Hardman. LODE: Linking Open Descriptions Of Events. In $4^{\text {th }}$ Asian Semantic Web Conference, 2009.

[13] R. Troncy, A. Fialho, L. Hardman, and C. Saathoff. Experiencing Events through User-Generated Media. In $1^{\text {st }}$ International Workshop on Consuming Linked Data, 2010.

[14] R. Troncy, B. Malocha, and A. Fialho. Linking Events with Media. In $6^{\text {th }}$ International Conference on Semantic Systems, 2010.

[15] T. Wang, T. Mei, X.-S. Hua, X.-L. Liu, and H.-Q. Zhou. Video Collage: A Novel Presentation of Video Sequence. In IEEE International Conference on Multimedia and Expo, 2007. 